\title{
VALOR ECONÔMICO DOS DANOS AMBIENTAIS DO RIO MEIA PONTE EM GOIÂNIA (GO)
}

Francis Lee Ribeiro*

Carlos Leão**

\section{Resumo}

O Rio Meia Ponte é um importante recurso hídrico do Estado de Goiás, mas sua qualidade natural está comprometida da nascente à foz. O ápice da poluição localiza-se em Goiânia, capital do estado, onde são desenvolvidas várias atividades produtivas às margens desse rio, notadamente a horticultura, cuja produção é encaminhada ao mercado. A irrigação das hortas com água poluída pode levar à contaminação dos produtos e acarretar prejuízos à saúde dos consumidores. A não-existência de mercados onde bens públicos, tal como a qualidade do Rio Meia Ponte, sejam transacionados implica o desconhecimento de um valor para essa categoria de bens. Tal valor é indicador da importância do nível de qualidade desse bem para o bem-estar da população, já que permite a estimativa dos danos decorrentes da poluição, ou dos benefícios gerados pela despoluição. Com o objetivo de estimar o valor dos danos decorrentes da poluição do Rio Meia Ponte para a população de Goiânia, adotou-se o Método de Avaliação Contingente, pelo qual se estima tal valor monetário com base nas preferências individuais reveladas no contexto de um mercado hipotético.

Palavras-chave: Valoração contingente, danos ambientais, Logit.

\section{Introdução}

Em 1997 empreendeu-se em Goiânia, Goiás, uma pesquisa com o objetivo de verificar como a degradação do Rio Meia Ponte era percebida pela população goianiense. Buscou-se também estimar qual o valor dos danos, em termos monetários, associado a tal degradação ambiental.

\footnotetext{
* Doutoranda em Economia Rural na UFV. E-mail: francisleerib@.bol.com.br

** Professor de Economia do Departamento de Ciências Sociais da UFG.

E-mail: cleao@fchf.ufg.br
} 
Naquele momento, as autoridades públicas municipais e estaduais não se mostraram atraídas pelo tema em estudo. Hoje, quatro anos após, a degradação do Rio Meia Ponte figura como tópico importante na agenda governamental, o que se deveu, em grande parte, à pressão exercida pela sociedade. Assim, os resultados da pesquisa constituem, hoje, informações relevantes para a análise de investimentos e do bem-estar social referentes à qualidade do Rio Meia Ponte.

No Brasil, a tendência dos últimos anos é de piora da qualidade da água e, particularmente no Estado de Goiás, a poluição hídrica é crescente em consequiência dos impactos ambientais provocados pelo crescimento das cidades e pelo aumento das atividades agrícolas e industriais. O Rio Meia Ponte é um importante recurso natural desse Estado, que, segundo a Femago (1990), está comprometido desde a nascente até a foz, sendo que o ápice da poluição ocorre no município de Goiânia. Esta é a capital do Estado e também o mais importante centro regional (populacional e econômico) e, conseqüentemente, também o espaço cristalizador dos impactos do desenvolvimento. Assim, considerou-se a importância da realização da pesquisa ainda que restrita apenas a essa cidade.

Conforme o Censo Industrial de Goiás dos estabelecimentos industriais do Estado de Goiás, 22,12\% concentravam-se na capital em 1985. Os 13 gêneros de indústrias de transformação considerados os mais poluentes estão presentes na cidade e muitos deles estão estabelecidos próximos aos cursos d'água, em especial, do Rio Meia Pontes e seus afluentes. Como poucas indústrias e também poucos hospitais possuem tratamento próprio de esgoto, a maioria deles faz seus despejos diretamente nos cursos d'água ou na rede pública de coleta. Extraoficialmente, diz-se que apenas $10 \%$ do esgoto coletado é tratado antes de ser despejado no Rio Meia Ponte, o mesmo que, a montante da cidade, é aproveitado para a captação de água para abastecimento urbano.

$\mathrm{O}$ desenvolvimento da horticultura às margens desse rio e de seus afluentes, dentro do município de Goiânia, agrava a poluição do Rio Meia Ponte. Em razão de a horticultura, atividade agrícola que pode ser desenvolvida em pequenas áreas, ser altamente dependente de grande consumo de água, um número expressivo de hortas localiza-se às margens dos cursos d'água. 
O mercado goianiense de hortaliças recebe a produção obtida nessas propriedades ribeirinhas que usam água poluída para irrigar as plantações. Embora esse mercado seja abastecido de hortaliças provenientes de outras zonas de produção ou, ainda, dos chamados "produtos limpos", os produtos de diversas fontes misturam-se no mercado, e o consumidor não tem certeza da origem do produto que adquire. Portanto, a proteção do Meia Ponte pode aumentar o bemestar dos indivíduos, que, embora não se relacionem diretamente com o bem, são beneficiários da restituição e conservação da qualidade natural das águas desse rio. Daí a determinação da população a ser pesquisada: os consumidores de hortaliças em Goiânia.

A obtenção de valores monetários para bens e serviços ambientais ainda constitui tópico de debates e polêmicas tanto na academia quanto fora dela, o que se deve, em grande parte, ao caráter público dessa categoria de bens. O Rio Meia Ponte é um bem de consumo coletivo, um bem público, que tem valor para a sociedade mas não tem mercado onde tal valor possa ser expresso. Assim, a teoria econômica neoclássica tem desenvolvido métodos alternativos para a obtenção de valores extramercado, considerando a importância deles na tomada de decisão relativa a esses bens. A mensuração do valor do Rio Meia Ponte é um indicador da importância que o nível de qualidade desse recurso tem para o bem-estar dos goianienses, pois permite a estimativa dos danos ambientais decorrentes da poluição, o que equivale aos benefícios gerados pela "despoluição".

Esse estudo teve como objetivo central obter uma estimativa monetária dos danos ambientais do Rio Meia Ponte para a população de Goiânia. Como suporte da política ambiental referente a esse recurso, objetivou-se mostrar que medidas relativas ao controle da poluição eram desejadas pela sociedade goianiense. Deve-se salientar que a estimativa de valores ambientais é exigida pelas agências internacionais de crédito (BID e BIRD) em estudos para a concessão de financiamentos Em muitas experiências com instrumentos econômicos, as políticas de meio ambiente de vários países, notadamente daqueles que integram a OCDE, a apuração dos valores dos danos ambientais é fundamental para a constituição das bases para o estabelecimento de valores para taxas. 
Além desta introdução, o presente artigo foi dividido em cinco seções antes de apresentar a conclusão. Na primeira seção define-se o "valor econômico total do meio ambiente", bem como as categorias que constituem esse valor. Na segunda seção é feita, em linhas gerais, a descrição da estrutura e da fundamentação teórica do método de avaliação contingente, adotado no presente estudo; apontam-se as principais críticas ao método e ainda se apresenta a técnica usada para a obtenção da disposição a pagar (DAP) dos indivíduos. A terceira seção apresenta o modelo analítico para os dados obtidos com a aplicação do método. A quarta demonstra como foi estruturada a pesquisa de campo, e a quinta seção traz os resultados obtidos com a pesquisa.

\section{O valor econômico do meio ambiente}

O valor econômico do meio ambiente é definido como:

VALOR ECONÔMICO TOTAL = Valor de Uso + Valor de Não-Uso

em que:

Valor de Uso = Valor de Uso Atual + Valor de Opção

O "valor de uso atual" na expressão (2) consiste de todos os modos correntes diretos em que um agente espera fazer uso físico do bem ambiental. Essa classe de benefícios também tem uma dimensão indireta criada quando as características do bem ambiental aumentam as atividades nas proximidades. O "valor de opção", ainda na expressão (2), é um valor desconhecido pois refere-se ao valor atribuído pelos indivíduos a um possível uso futuro do bem. Na expressão (1), além do "valor de uso" já definido, apresenta-se o "valor de não-uso" (valor intrínseco, ou, ainda, valor de existência). Esse é definido como o valor que o meio ambiente tem por si mesmo, ou seja, independe de usos atuais ou futuros (PEARce e Turner, 1990).

O ponto fundamental da conceituação de "valor econômico do meio ambiente" é a definição de um "valor total", A definição dessas categorias de valor significa um reconhecimento da existência de elementos da globalidade espacial, intertemporal e extra-econômica do problema ambiental. $\mathrm{O}$ valor de uso delimitaria o universo dos indivíduos 
envolvidos; o valor de opção refere-se às diferentes possibilidades futuras e o valor de existência trataria dos aspectos extra-econômicos. Enquanto os métodos indiretos de valoração de bens públicos e, mais específicamente, de bens ambientais só podem captar parte do "valor total", isto é, o "valor de uso", acredita-se genericamente, embora não de forma absolutamente consensual, que o Método de Avaliação Contingente ${ }^{1}$ (MAC) é capaz de medir benefícios que incluem uma dimensão de nãouso (Mitchell e CARson, 1989).

\section{O método de avaliação contingente}

Apesar de se reconhecer que não há mercados para a determinação de valores ambientais de modo condizente ao seu método, os neoclássicos procuram encontrá-los no mesmo princípio que orientaria a formação de preços de mercado: a agregação das preferências individuais. Assim é no intuito de determinar quantitativamente as externalidades e sua função de degradação, a partir desses valores de uso, de opção e de existência, que a economia neoclássica procura desenvolver metodologias de mensuração que visam captar as preferências e os "preços-sombra" correspondentes a tais valores.

O Método de Avaliação Contingente estima o valor monetário de bens e serviços ambientais com base em preferências expressas por consumidores potenciais, relativas à disponibilidade e aos acréscimos ou decréscimos na qualidade desses bens e serviços. Essa valoração é fundamentada em dois conceitos fundamentais: um baseado na "disposição a pagar" (DAP) e outro na "disposição a aceitar" (DAA). Tem-se que a DAA e a DAP são indicadores monetários de preferências e seus conceitos estão relacionados com medidas de bem-estar. Nos mercados, o comportamento dos indivíduos revela a curva de demanda ordinária (marshalliana), mas trata-se aqui de bens para os quais não existem mercados. O MAC objetiva contornar ou eliminar esse problema perguntando diretamente aos indivíduos qual sua DAP ou DAA para uma alteração na provisão de um bem público. Assim, os indivíduos revelam a curva de demanda compensada, e as medidas de bem-estar geradas pelo método são as medidas hicksianas. 
O MAC consiste num processo de perguntas aos indivíduos sobre sua DAP ou DAA para garantir um benefício ou para abrir mão dele. $\mathrm{O}$ processo de perguntas se dá no âmbito de um mercado hipotético construído pelo pesquisador no próprio contexto da pesquisa. $\mathrm{O}$ formato da questão de valoração contingencial colocada no plano da entrevista irá condicionar o tipo de medida de bem-estar que se pode obter do processo (medidas de excedente de equivalência ou excedente de compensação).

O interesse pela valoração de bens públicos e, mais particularmente, de bens ambientais tem propiciado uma grande evolução do método. Contudo, ele é objeto de várias críticas e objeções, que, em última instância, referem-se ao seu caráter hipotético. Num mercado hipotético a presença de vieses pode interferir significativamente nos resultados obtidos. Os vieses que mais frequientemente podem estar presentes na aplicação do MAC podem ser classificados, conforme sua natureza, nas seguintes categorias: viés estratégico, viés do desenho do questionário, viés hipotético, viés operacional, viés de protesto.

O uso de mercados hipotéticos para valoração de bens públicos inclui uma variedade de técnicas, sendo que as mais usadas recentemente podem ser subdivididas em formas iterativas e não-iterativas. Neste estudo adotou-se o mecanismo referendum, que é a técnica correntemente mais empregada e que, dentro do intervalo de valores da pesquisa, apresenta apenas um valor aleatório a cada entrevistado. Essa técnica pode ser descrita da seguinte forma: tomado do intervalo de valores que constituem a pesquisa, um valor é apresentado ao entrevistado que revelará sua disposição a pagar para garantir um benefício ou evitar um dano associado ao bem ou serviço ambiental pesquisado.

\section{Função diferença de utilidades ${ }^{2}$}

A pergunta "Você estaria disposto a pagar mensalmente a quantia de R\$ p para que o Rio Meia Ponte fosse despoluído e conservado?" admite que o indivíduo derive sua utilidade do consumo de hortaliças e da renda monetária. É bom lembrar que esse tipo de pergunta leva a uma medida de excedente de compensação. Seja então, a seguinte função utilidade: ${ }^{3}$ 
$\mathrm{u}(\mathrm{j}, \mathrm{y} ; \mathrm{s})$

em que:

$\mathrm{j}$ = variável binária; $\mathrm{j}=1$ significa "rio protegido" e $\mathrm{j}=0$ significa "rio poluído";

$\mathrm{y}=$ renda;

$\mathrm{s}=$ vetor que representa outros atributos do indivíduo que possam afetar sua preferência. Portanto:

$\mathrm{u}_{1}=\mathrm{u}(1, \mathrm{y} ; \mathrm{s})$,

se o indivíduo pode consumir hortaliças “saudáveis” (com rio protegido).

$\mathrm{u}_{0}=\mathrm{u}(0, \mathrm{y} ; \mathrm{s})$,

se o indivíduo não pode consumir hortaliças "saudáveis" (com o rio poluído)

$\mathrm{u}_{1}$ e $\mathrm{u}_{0}$ são variáveis aleatórias com certa distribuição de probabilidade e com médias v $(0, y$; s $)$ e v $(1, y$; s). Portanto, as utilidades são escritas como:

$u(j, y ; s)=v(j, y ; s)+e_{j ;} j=0,1$

em que $e_{0}$ e e $e_{1}$ são variáveis aleatórias idênticas e independentemente distribuídas, com média zero e variância finita,

Os indivíduos respondem positivamente se:

$v(1, y-p ; s)-v(0, y ; s)^{3} e_{0}-e_{1}$

em que $p$ é a quantia que o indivíduo pagaria mensalmente para ter o bem "hortaliças saudáveis" (rio protegido).

Enquanto o indivíduo tem certeza quanto à escolha que maximiza sua utilidade, sob o ponto de vista do econometrista, a resposta do indivíduo é uma variável aleatória cuja distribuição de probabilidade é dada por:

$\mathrm{P}_{1}=\mathrm{P}_{\mathrm{r}}\{\mathrm{o}$ indivíduo aceita pagar $\}$ 
$\mathrm{P}_{1}=\mathrm{P}_{\mathrm{r}}\left\{\mathrm{v}(1, \mathrm{y}-\mathrm{p} ; \mathrm{s})+\mathrm{e}_{1}{ }^{3} \mathrm{v}(0, \mathrm{y} ; \mathrm{s})+\mathrm{e}_{0}\right\}$

$\mathrm{P}_{1}=\mathrm{P}_{\mathrm{r}}\{\Delta \mathrm{v} \geq \delta\}$

em que:

$\Delta \mathrm{v}=\mathrm{v}(1, \mathrm{y}-\mathrm{p} ; \mathrm{s})-\mathrm{v}(0, \mathrm{y} ; \mathrm{s})$

$\delta=e_{0}-e_{1}$

Conseqüientemente:

$\mathrm{P}_{0}=\mathrm{P}_{\mathrm{r}}\{$ o indivíduo não aceita pagar $\}$

$\mathrm{P}_{0}=1-\mathrm{P}_{1}$

Dado que $\delta=\mathrm{e}_{0}-\mathrm{e}_{1}$ faz $\mathrm{F}_{\delta}($.$) ser a função densidade acumulada$ de $\delta$, a probabilidade de que o indivíduo esteja disposto a pagar $\mathrm{R} \$ \mathrm{p}$ pode ser escrita como:

$\mathrm{P}_{1}=\mathrm{F}_{\delta}(\Delta \mathrm{v})$

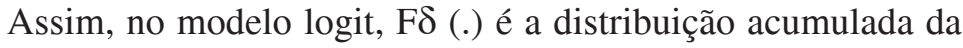
função logística padrão e $\Delta v$ é a Função Diferença de Utilidades, tal como dada na equação (11), e o modelo estatístico das respostas binárias pode ser interpretado como o resultado de uma escolha que maximiza utilidade. Logo, a forma funcional relevante a ser especificada é a de $\Delta \mathrm{v}$ (HANEMANN, 1984), que propõe que primeiro seja especificada a função utilidade, obtendo-se a forma funcional de $\Delta \mathrm{v}$ por manipulação.

Para calcular uma medida, baseada na utilidade, do valor monetário dos benefícios atribuídos à disponibilidade do bem, estima-se uma quantia $\mathrm{R} \$$ p que satisfaça a igualdade:

$\mathrm{v}\left(1, \mathrm{y}-\mathrm{p}^{*} ; \mathrm{s}\right)-\mathrm{v}(0, \mathrm{y} ; \mathrm{s})=\delta$

$\mathrm{R} \$ \mathrm{p}$ é o valor que faria um indivíduo permanecer indiferente entre escolher hortaliças não-saudáveis (rio poluído) e renda total y, ou hortaliças saudáveis (rio protegido) e renda menor $\left(\mathrm{y}-\mathrm{p}^{*}\right)$, sendo $\mathrm{p}^{*} \mathrm{o}$ valor a ser pago para a obtenção do benefício. Se $\delta$ tem uma distribuição logística padronizada, a mediana e a média são iguais a zero. Então, o 
valor $\delta=0$ está associado ao ponto de indiferença, sendo $\mathrm{F}_{\delta}(0)=0,5$. Para $\Delta v=\delta=0$, o indivíduo estaria indiferente entre proteger ou não o bem, e o valor médio de p é considerado como aquele que o indivíduo estaria disposto a pagar por ele (Aguirre e FAria, 1996). Assim, tem-se:

$\operatorname{Pr}\{\Delta \mathrm{v}=\delta=0\}=\mathrm{F}_{\delta}(\Delta \mathrm{v}=0)=0,5$

Portanto, no modelo logit, $\mathrm{p}^{*}$ satisfaz a condição $\Delta \mathrm{v}\left(\mathrm{p}^{*}\right)=0$

\section{Estruturação da pesquisa de campo}

Embora o bem público particular nesta pesquisa tenha uma localização mais abrangente (35 municípios goianos) que o seu espaço interno ao município de Goiânia, justificou-se que essa região metropolitana é caracterizada como o mais importante e o maior centro poluidor do Rio Meia Ponte. Assim, poder-se-iam tomar todos os residentes goianienses na determinação da população da pesquisa.

A associação do Rio Meia Ponte com a horticultura praticada nas suas margens definiu uma relação entre a população urbana global e o recurso em questão. Determinou-se assim que a população da pesquisa são os consumidores de hortaliças em Goiânia. Os consumidores são identificados no mercado. Optou-se por realizar a pesquisa nas feiras livres sob a consideração de que elas são distribuídas em bairros estratégicos nas regiões urbanas, recebem consumidores dos diferentes segmentos sociais e comercializam produtos oriundos de diversas regiões produtoras.

Realizou-se preliminarmente uma pesquisa-piloto. Essa fase da pesquisa constitui-se de 53 observações e, com base nos resultados, foram escolhidos oito valores com os quais foram construídos três intervalos de disposição a pagar. São eles:

\begin{tabular}{|c|c|c|}
\hline Intervalo A: & $\mathrm{R} \$ 3,00$ & $\mathrm{R} \$ 9,00$ \\
\hline Intervalo B: & $\mathrm{R} \$ 18,00$ & $\mathrm{R} \$ 24,00$ \\
\hline Intervalo C: & $\mathrm{R} \$ 30,00$ & $\mathrm{R} \$ 38,00$ \\
\hline
\end{tabular}

Cada questionário foi preenchido com um valor de um intervalo, gerando, então, nove tipos diferenciados de questionários que foram apresentados aleatóriamente aos indivíduos entrevistados. 


\section{Estimação da disposição a pagar}

Seja a seguinte função utilidade proposta por Hanemann (1984):

$v(j, y ; s)=a_{j}(s)+b y j=0,1 ; b>0$

A função acima especificada é linear em y e, portanto, a utilidade marginal da renda é constante, implicando que as probabilidades associadas com a variável discreta de escolha independem da renda do indivíduo. Ocorrem, assim, apenas os efeitos-substituição e não os efeitosrenda (HANEMANN, 1989).

Aplicando em (19) a definição de Função Diferença de Utilidades dada em (11), segue que:

$$
\begin{aligned}
& \Delta \mathrm{v}=\mathrm{a}_{1}(\mathrm{~s})+\mathrm{b}(\mathrm{y}-\mathrm{p})-\mathrm{a}_{0}(\mathrm{~s})-\mathrm{by} \\
& \Delta \mathrm{v}=\left[\mathrm{a}_{1}(\mathrm{~s})-\mathrm{a}_{0}(\mathrm{~s})\right]-\mathrm{b} \mathrm{p} \\
& \Delta \mathrm{v}=\left(\mathrm{a}_{1}-\mathrm{a}_{0}\right)-\mathrm{b} \mathrm{p} \\
& \Delta \mathrm{v}=\mathrm{a}-\mathrm{b} \mathrm{p}
\end{aligned}
$$

Substituindo (23) em (15) tem-se que o modelo estatístico de escolha é: ${ }^{4}$

$\mathrm{P}_{1}=\mathrm{F}_{\delta}(\mathrm{a}-\mathrm{b} \mathrm{p})$

em que:

$\mathrm{a}=\mathrm{a}_{1}-\mathrm{a}_{0}$

Porém $\mathrm{a}_{1}$ e $\mathrm{a}_{0}$ não podem ser identificados a partir dos dados, sendo que apenas a diferença entre eles é identificável (HANEMANN, 1984).

Na seqüência, a função determinada em (24) é estimada pelo modelo logit, cuja forma funcional utilizada é linear, e a equação ajustada pelo método de máxima verossimilhança foi definida por:

$\Delta v=\alpha+\beta_{1}$ PREÇO $+\beta_{2}$ RENFA $+\beta_{3}$ ORIG $+\beta_{4}$ PROT

sendo: 
$\Delta \mathrm{v}=$ variável dependente binária com valor 0 para resposta NÃO e 1 para resposta SIM;

PREÇO = valores monetários de disposição a pagar

RENFA $=$ intervalos de renda familiar, quais sejam:

\begin{tabular}{|l|l|l|l|}
\hline 1 & 1 salário mínimo & 4 & 11 a 15 salários mínimos \\
\hline 2 & 2 a 5 salários mínimos & 5 & 16 a 20 salários mínimos \\
\hline 3 & 6 a 10 salários mínimos & 6 & mais de 20 salários mínimos \\
\hline
\end{tabular}

$\mathrm{ORIG}=$ dummy com valores 0 e 1 , respectivamente representando os indivíduos que não têm certeza da origem das hortaliças que adquirem e caso contrário;

PROT $=$ variável indicativa de vieses nas respostas de valoração contingente, sendo 0 para a ausência de viés e:

\begin{tabular}{|c|l|c|l|}
\hline 1 & motivos financeiros & 5 & $\begin{array}{l}\text { discorda em pagar para obter } \\
\text { a melhoria }\end{array}$ \\
\hline 2 & falta de interesse & 6 & não entendeu a pergunta \\
\hline 3 & $\begin{array}{l}\text { discorda da forma de } \\
\text { pagamento }\end{array}$ & 7 & precisa de tempo para pensar \\
\hline 4 & $\begin{array}{l}\text { não acredita que pagando } \\
\text { ocorrerá a melhoria }\end{array}$ & 8 & outros \\
\hline
\end{tabular}

As estimativas dos coeficientes são as seguintes:

$$
\begin{aligned}
& \hat{\Delta} \mathrm{v}=0,939188-0,039731 \text { PREÇO + 0,293430 RENFA+ 0,367873 ORIG - } \\
& 0,416171 \text { PROT }
\end{aligned}
$$

A variável PREÇO tem coeficiente negativo e significativo. Tomando-se o valor médio o seu efeito marginal sobre a probabilidade de resposta SIM, a valoração contingencial é de -0,0071852, isto é, o aumento de uma unidade de valor (em termos monetários), ceteris paribus, a partir do preço médio $(\mathrm{R} \$ 22,55)$, reduz a probabilidade de ocorrência do evento SIM em $0,72 \%$. 
PARÂMETROS ESTIMADOS DO MODELO LOGIT PARA A "DISPOSIÇÃO A PAGAR" PELA DESPOLUIÇÃO DO RIO MEIA PONTE

EM GOIÂNIA, 1997

\begin{tabular}{|c|c|c|c|c|}
\hline \multicolumn{5}{|c|}{ LOGIT // VARiável Dependente É $\Delta \mathrm{v}$} \\
\hline \multicolumn{5}{|c|}{ AMOSTRA: 1.505} \\
\hline \multicolumn{5}{|c|}{ OBSERVAÇÕES INCLUIDAS: 505} \\
\hline \multicolumn{5}{|c|}{ CONVERGÊNCIA APÓS 3 ITERAÇÕES } \\
\hline VARIÁVEL & Coeficiente & ERRO PADRÃO & T-S $\mathrm{S}_{\text {TUDENT }}$ & РRов \\
\hline $\mathrm{C}$ & 0,939188 & 0,333564 & 2,815620 & 0,0051 \\
\hline PREÇO & $-0,039731$ & 0,009255 & $-4,293113$ & 0,0000 \\
\hline RENFA & 0,293430 & 0,084132 & 3,487730 & 0,0005 \\
\hline ORIG & 0,367873 & 0,261318 & 1,407757 & 0,1598 \\
\hline РRот & $-0,416171$ & 0,043213 & $-9,630600$ & 0,0000 \\
\hline \multicolumn{5}{|c|}{$\begin{array}{ll}\text { LOG DA VEROSIMILHANÇA } & -302,7856\end{array}$} \\
\hline \multicolumn{3}{|c|}{ Ове сом $\mathrm{Y}=1$} & \multicolumn{2}{|l|}{226} \\
\hline \multicolumn{3}{|c|}{ Oвs сом $\mathrm{Y}=0$} & \multicolumn{2}{|l|}{279} \\
\hline
\end{tabular}

\begin{tabular}{|l|l|c|c|}
\hline VARIÁVEL & MÉDIA TODOS & MÉDIA Y $=1$ & MÉDIA Y $=0$ \\
\hline C & 1,000000 & 1,000000 & 1,000000 \\
\hline PREÇO & 22,55050 & 20,00000 & 24,61649 \\
\hline RENFA & 3,229703 & 3,336283 & 3,143369 \\
\hline ORIG & 0,192079 & 0,221239 & 0,168459 \\
\hline PROT & 3,312871 & 1,827434 & 4,516129 \\
\hline IRV $=0,78$ & (Índice de Razão Verossimilhança) \\
\hline
\end{tabular}

Fonte: Resultados da pesquisa.

A variável RENFA apresenta coeficiente positivo e significativamente correlacionado à resposta SIM, implicando que, ceteris paribus, aumentos na renda aumentam a probabilidade de respostas afirmativas, sendo que o seu efeito marginal estimado é de 5,31\%.

A variável ORIG também apresenta coeficiente significativo e positivo, indicando que quando os indivíduos não têm certeza da origem dos produtos que adquirem maior é a probabilidade de resposta SIM. O efeito marginal dessa variável é de 6,65\%. 
A variável PROT também é significativa e seu coeficiente é negativo, indicando que a presença de vieses interfere negativamente na valoração contingencial. Seu efeito marginal foi estimado em 7,53\%.

Os resultados econométricos até aqui obtidos, a partir da estimação da "função diferença de utilidades", representam apenas a probabilidade associada à resposta SIM da avaliação contingente. Para obtenção da medida monetária de bem-estar deve-se igualar a função $\Delta \mathrm{v}$ estimada a zero e resolvê-la para o preço.

Seja a função definida em (20) a (23):

$\Delta v=a-b p$

Fazendo $\Delta v=0$ :

$\mathrm{a}-\mathrm{b} \mathrm{p}=0$

Resolvendo para p, obtém-se:

$p^{*}=\frac{a}{b}$

$\hat{\alpha}+\hat{\beta}_{2}(\overline{R E N F A})+\hat{\beta}_{3}(\overline{O R I G})-\hat{\beta}_{4}(\overline{P R O T})$

Tal como definido em (16) à (18), a partir de (29) obtém-se o valor médio (e mediano) de p denotado por $\mathrm{p}^{*}$, calculado em função dos coeficientes do modelo estatístico discreto de escolha. $\mathrm{O}$ valor do dano ou benefício individual é dado por:

Dano Individual $=\frac{\alpha^{*}}{\beta *}$

Sendo $\mathrm{a}^{*}$ a estimativa dos coeficientes que representam $a \mathrm{e} \mathrm{b}$ as estimativas de $b$ em (29), então:

$\alpha^{*}=$

$\beta^{*}=\hat{\beta}_{1} P R E C ̧ O$ 
Substituindo as variáveis RENFA, ORIG e PROT, apresentadas em (31), por seus respectivos valores médios (média “todos” do Quadro 1), e usando o valor estimado dos parâmetros e o coeficiente estimado da variável preço em (32), têm-se:

$\alpha^{*}=0,578819591$

$\beta^{*}=-0,039731$

Dano Individual $=14,56846269=\mathrm{R} \$ 14,57$

O valor estimado $(\mathrm{R} \$ 14,57)$ representa o dano (ou perda) mensal por indivíduo, proveniente da ausência ou deficiência no controle da poluição do Rio Meia Ponte em Goiânia, ou, expressando de outro modo, esse valor representa o benefício individual mensal a ser obtido com a recuperação e controle da qualidade do Rio Meia Ponte

\section{Conclusões}

A pesquisa foi muito bem recebida pelos indivíduos entrevistados e pôde-se perceber que a população pesquisada tinha conhecimento do quadro geral de degradação do Rio Meia Ponte, das causas e das possíveis conseqüências. Ainda foi constatada a identificação de classes de benefícios pelos entrevistados, sendo que o ponto mais interessante observado é que o Rio Meia Ponte tem um "valor de não-uso" para os goianienses.

A validade do resultado obtido, ou seja, do valor monetário estimado, depende em grande medida da estruturação da pesquisa que gerou os dados utilizados nas estimações. Analisou-se que, quanto à amostragem, a definição da população representada contemplou a possibilidade de apuração de um "valor total", não se restringindo apenas ao "valor de uso". Dessa forma, pode-se considerar o "valor de nãouso", não limitando o método de avaliação contingente e podendo-se captar sua característica mais interessante. Quanto ao mercado hipotético, o cenário apresentado restringiu-se à problemática do consumo de hortaliças ainda que a questão de valoração tenha sido colocada de maneira ampla com base nos benefícios obtidos com a despoluição do 
Rio Meia Ponte. O nível de conscientização da população pesquisada e os procedimentos de campo garantiram a obtenção do "valor total" dos benefícios ou danos.

O valor estimado é referente a todos os benefícios que o indivíduo pode derivar do uso físico do Rio Meia Ponte, direta ou indiretamente, e ainda da própria "existência" ou "não-uso" do bem. Contudo, ainda que os vieses tenham sido considerados no processo de estimação, a presença deles influenciou a pesquisa e a aplicação do método. Especificamente, quanto ao viés de protesto, tem-se que a credibilidade dos indivíduos na administração pública e em seus responsáveis foi uma variável muito relevante no processo de valoração. Os formuladores de políticas devem estar cientes desse fato.

Concluindo pela validade do valor estimado e apresentando-o como "valor total" têm-se que o estado de degradação do Rio Meia Ponte, conforme se apresentava em julho de 1997, implicava perda de bem-estar para a população de Goiânia, o que sugeria o interesse dessa mesma população por uma política de controle da poluição e conservação do rio. Tal valor constitui, hoje, uma variável importante nas análises de custo-benefício de programas e projetos para a revitalização do Meia Ponte e seus afluentes, além de ser referência para a cobrança do uso da água bruta e para a elaboração de políticas baseadas em instrumentos de mercado. Considerando a crise fiscal por que passa o Estado brasileiro, cujas tentativas de ajuste têm acarretado um desmantelamento do próprio aparelho estatal - o que se constata ao se observarem os problemas nacionais da regulação, tal como a falta de coordenação entre os vários órgãos da área (de distintos níveis de competência) e, notadamente, a carência de recursos financeiros e humanos nas agências ambientais , fica evidente a oportunidade de discutir a introdução de instrumentos econômicos na política ambiental brasileira. ${ }^{5}$

\section{Abstract}

The Meia Ponte river is an important hydric resource of the State of Goiás, but its quality has been thouroghly jeopardized, with the worst polluted site being Goiânia, the state's capital. Several productive activities are developed along the margins of the river, notably horticulture, whose production is taken to the market. The irrigation of the vegetable gardens with polluted water can 
lead to contamination of the produce consequently causing damages to the consumers' health. The lack of markets where public goods, such as Meia Ponte river's quality, could be traded reflects a lack of knowledge of its value. Such value is indicative of the importance of this goods's quality level to the well being of the population, since it allows to estimate the damages caused by pollution or the benefits generated by depolluting. In order to estimate the financial cost of Meia Ponte river's pollution damage to the Goiânia population, the Contingent Valuation Method has been adopted. Through this method, such cost is estimated based on the individual preferences expressed within a context of a hypothetical market.

Key words: Contigent voluation, environmental damage, Logit.

\section{Notas}

1. Na última década, entre 18 projetos de ampliação de infra-estrutura que foram aprovados pelo BID (Banco Interamericano de Desenvolvimento), 13 empregaram o MAC na estimativa de benefícios.

2. Modelo proposto por Hanemann (1984).

3. A notação utilizada seguiu Aguirre e Faria (1996).

4. O vetor s foi suprimido porque $a$ e $b$ são funções de s.

5. O interesse crescente por instrumentos econômicos se justifica tanto pelo aspecto ambiental quanto pelo fiscal; os mecanismos de mercados são considerados mais adequados em condições de baixo crescimento econômico, déficits fiscais e inquietações sobre a competitividade internacional.

\section{Referências}

Aguirre, A.; FAria, D. M. C. P. Avaliação contingente de investimentos ambientais: um estudo de caso. Estudos Econômicos, São Paulo: IPEA, v. 26, n. 1. p. 85-109. jan./abr. 1996.

Femago. Fundação Estadual do Meio Ambiente. Departamento de Análises Ambientais. IQA: índice de qualidade das águas do RMP (1988 a 1990). Goiânia: Femago, 1990.

Hanemann, W. M. Welfare evaluations in contingent valuation experiments with discret responses. American Journal of Agricultural Economics, n. 66, p. 332-341, 1984. 
. Welfare evaluations in contingent valuation experiments with discret responses: reply. American Journal of Agricultural Economics, n. 71, p. 1057-1061, 1989.

Mitchell, R. C.; CARson, R. T. Using surveys to value public goods: the contingent valuation method. Washington, D. C.: Resources for the Future, 1989.

Pearce, D. W.; Turner, R. K. Economics of natural resources and environment. Londres: Harvaster, 1990. 
